\title{
Nanofibrous, Emulsion-Templated Syndiotactic Polystyrenes with Superhydrophobicity for Oil Spill Cleanup
}

Haoguan Gui, ${ }^{a, b}$ Tao Zhang, ${ }^{* a}$ and Qipeng Guo ${ }^{* b}$

${ }^{a}$ College of Textile and Clothing Engineering, Soochow University, Suzhou 215123,

China.

${ }^{b}$ Institute for Frontier Materials, Deakin University, Locked Bag 20000, Geelong, Victoria 3220, Australia.

E-mail:

zhang.tao@suda.edu.cn (T. Zhang)

qguo@,deakin.edu.au (Q. Guo) 


\section{Figures}

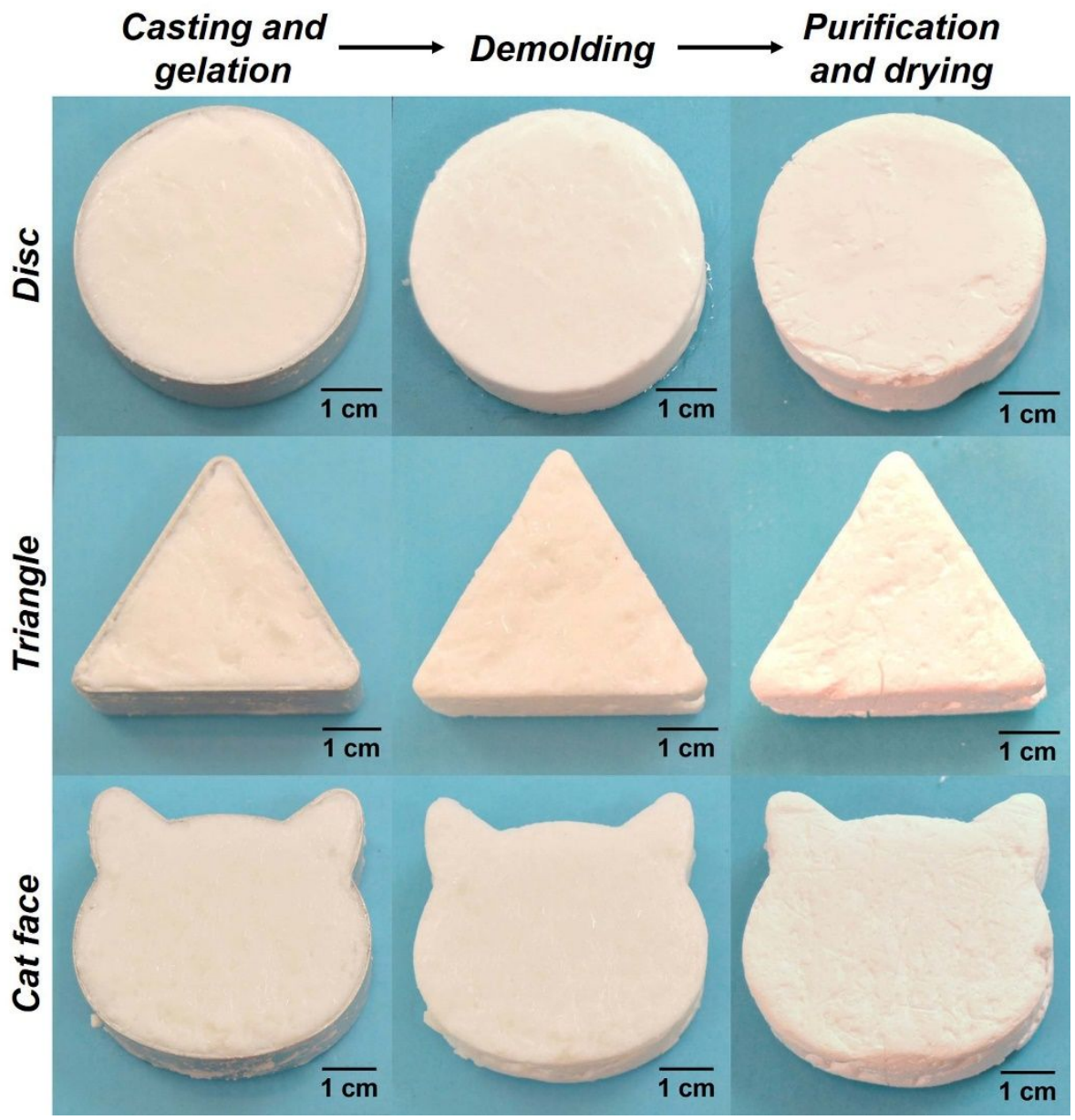

Figure S1. Preparation of the sPS- $X-Y$ with controllable shapes (disc, triangle, and cat face). 

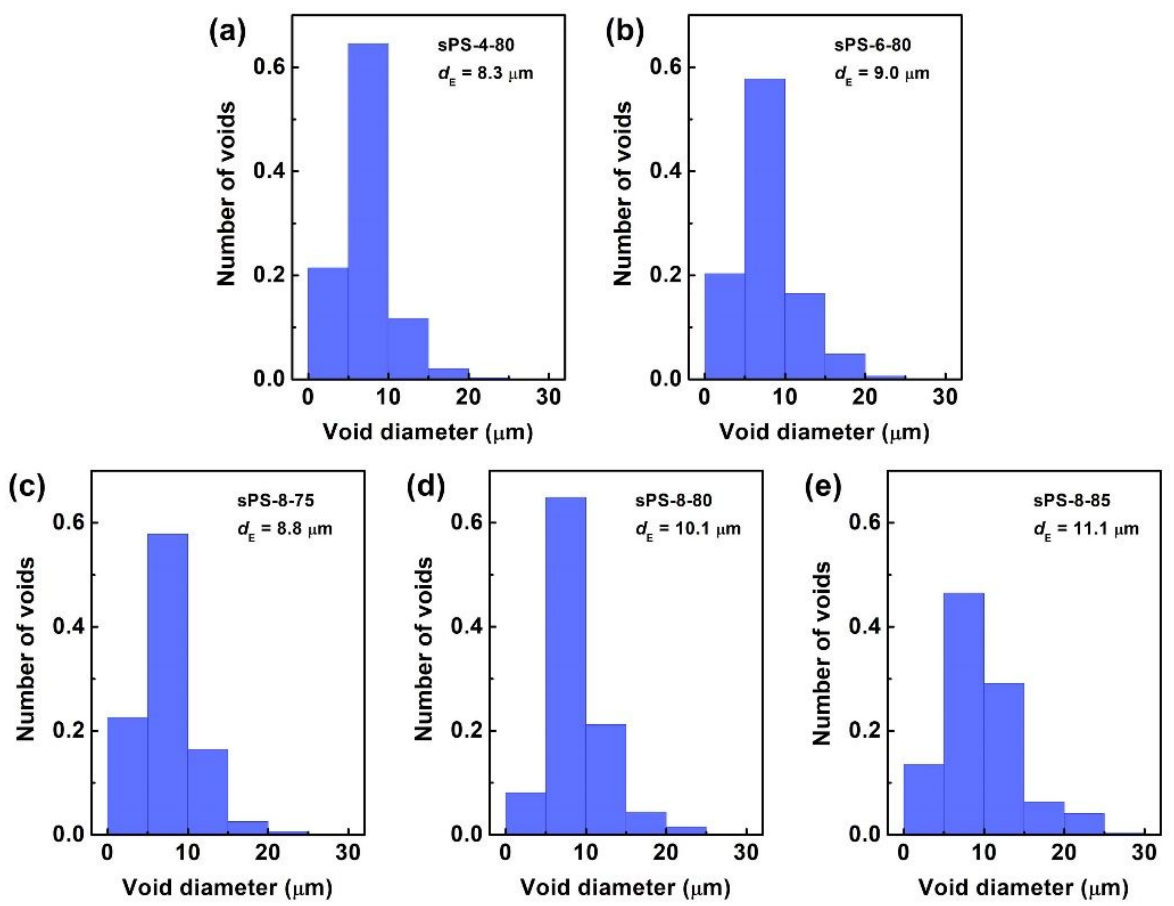

Figure S2. Emulsion-templated void diameter distributions of the sPS- $X-Y$ (determined from SEM micrographs): (a) sPS-4-80; (b) sPS-6-80; (c) sPS-8-75; (d) sPS-8-80; and (e) sPS-8-85.
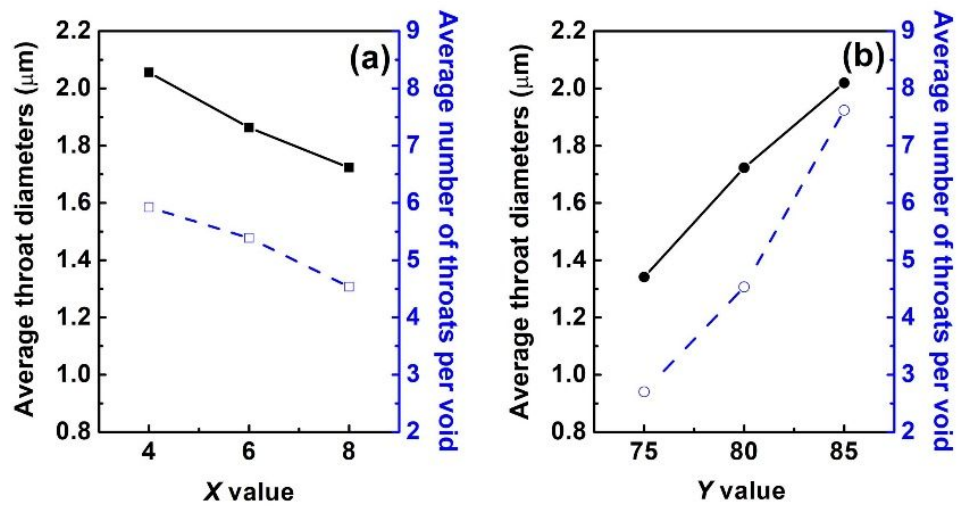

Figure S3. The average diameter of pore throats and the number of pore throats per emulsion-templated void within (a) the sPS- $X-80$ and (b) the sPS-8- $Y$. 

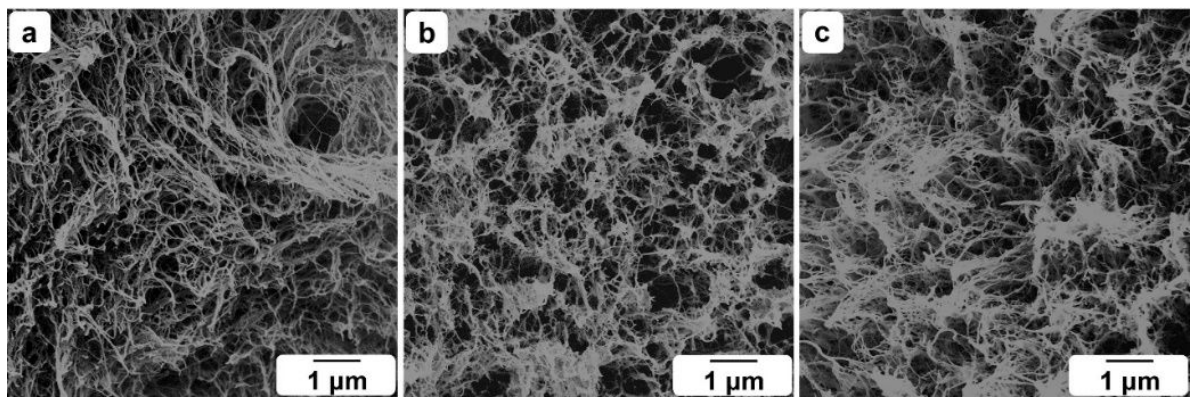

Figure S4. SEM micrographs of the sPS- $X$-bulk: (a) sPS-4-bulk, (b) sPS-6-bulk, and (c) sPS-8-bulk.
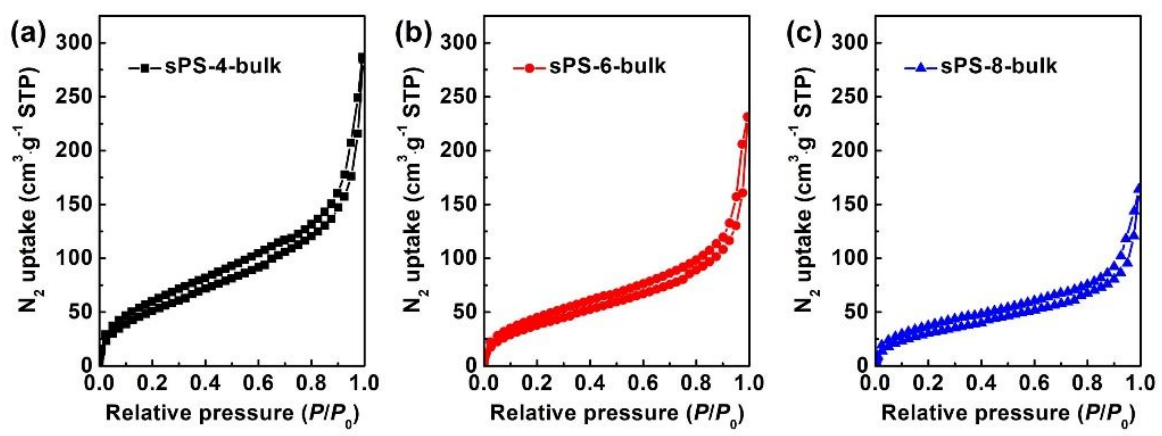

Figure S5. Nitrogen absorption-desorption isotherms of the sPS- $X$-bulk: (a) sPS-4-bulk, (b) sPS-6-bulk, and (c) sPS-8-bulk.
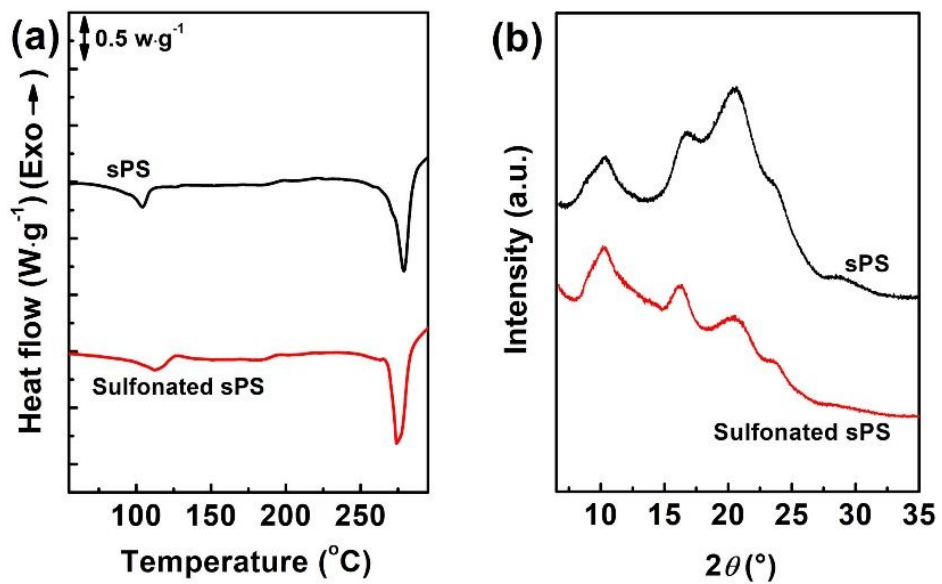

Figure S6. (a) DSC thermograms and (b) XRD pattern of the pure sPS and SsPS. 


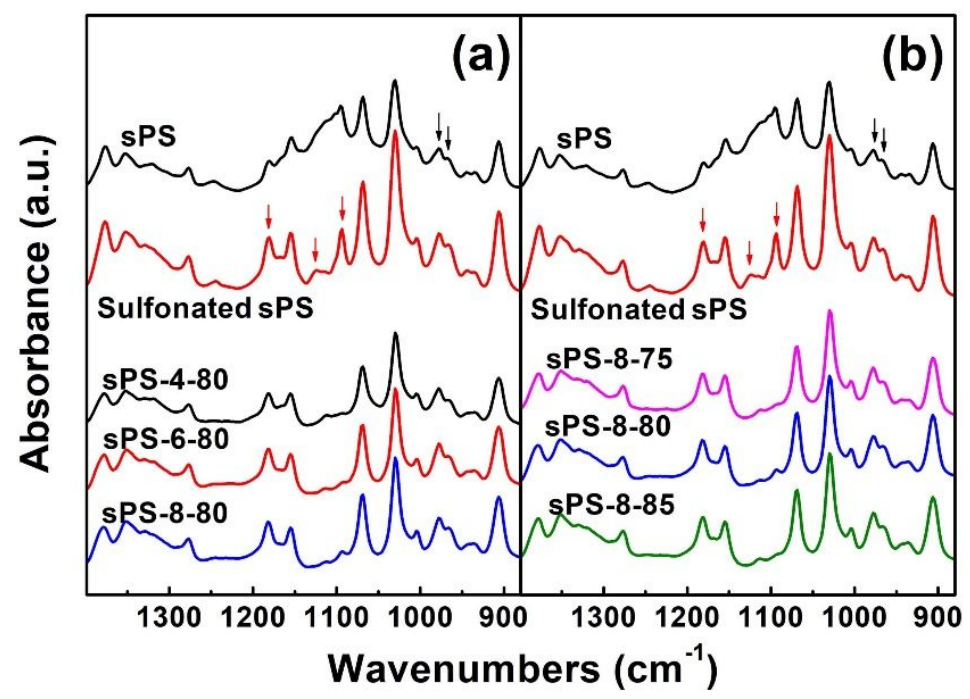

Figure S7. FTIR spectra $\left(1400 \sim 880 \mathrm{~cm}^{-1}\right)$ of sPS, SsPS and (a) the sPS- $X-80$ and (b) the sPS-8- $Y$.

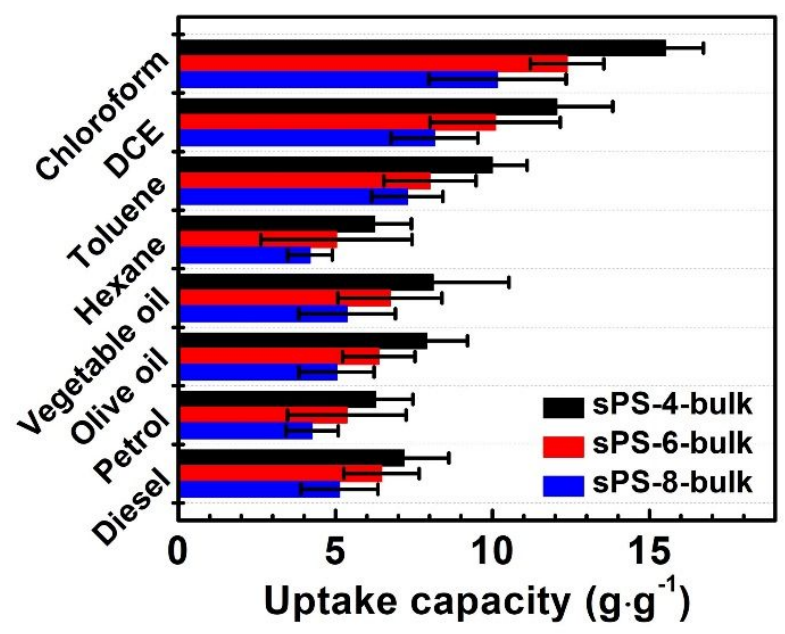

Figure S8. Equilibrium uptakes of various liquids within the sPS- $X$-bulk. 


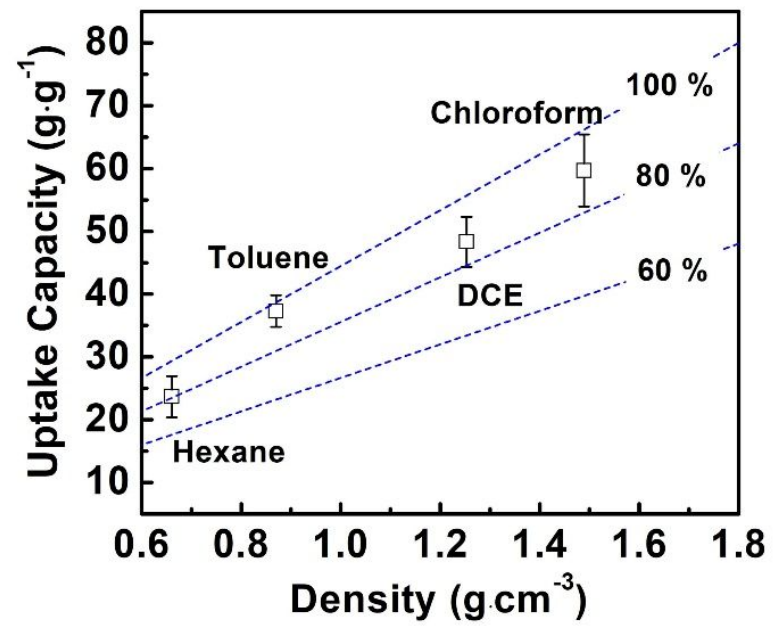

Figure S9. Equilibrium uptakes of various organic solvents within sPS-8-80 (dotted lines: standard uptakes when $60 \%, 80 \%$ and $100 \%$ of the pore volume are filled). 


\section{Table}

Table S1. A comparison in the liquid uptakes $\left(\mathrm{g} \cdot \mathrm{g}^{-1}\right)$ of typical HIPE-based absorbents reported in literatures to those of the sPS in the current work.

\begin{tabular}{|c|c|c|}
\hline Absorbents ${ }^{a}$ & $\begin{array}{l}\text { Absorbed solvents and the corresponding equilibrium } \\
\text { mass uptakes }\left(\mathrm{g} \cdot \mathrm{g}^{-1}\right)\end{array}$ & Ref. \\
\hline HIPE xerogels & $\begin{array}{l}\text { Hexane (19.8), toluene (22.3), dichloroethane (22.6), } \\
\text { chloroform (32.2), vegetable oil (24.2), petrol (21.2), } \\
\text { diesel (23.8), engine oil (31.5) and crude oil (15.1) }\end{array}$ & 1 \\
\hline PBA polyHIPE & $\begin{array}{l}\text { Toluene }(\sim 28) \text {, dichloroethane }(\sim 32) \text {, chloroform }(\sim 38) \text {, } \\
\text { petrol }(\sim 12) \text {, diesel }(\sim 15) \text { and engine oil }(\sim 14)\end{array}$ & 2 \\
\hline $\begin{array}{l}\text { PBMA } \\
\text { polyHIPE }\end{array}$ & $\begin{array}{l}\text { Hexane (3.86), kerosene (8.17) and used transformer oil } \\
(4.98)\end{array}$ & 3 \\
\hline $\begin{array}{l}\text { Hybrid } \\
\text { polyHIPE }\end{array}$ & Hexane (20.0), petrol (16.1), and kerosene (10.6) & 4 \\
\hline $\begin{array}{l}\text { Switchable } \\
\text { polyHIPE }\end{array}$ & $\begin{array}{l}\text { Hexane }(\sim 6), \text { toluene }(\sim 10) \text {, chloroform }(\sim 18) \text {, edible } \\
\text { oil }(\sim 9) \text {, petrol }(\sim 8) \text {, pump oil }(\sim 11) \text { and crude oil }(\sim 10)\end{array}$ & 5 \\
\hline $\begin{array}{l}\text { Melamine-based } \\
\text { polyHIPE }\end{array}$ & $\begin{array}{l}\text { Hexane }(\sim 16) \text {, chloroform }(\sim 35) \text {, mineral oil }(\sim 19) \text {, } \\
\text { paraffin oil }(\sim 21) \text { and silicon oil }(\sim 22)\end{array}$ & 6 \\
\hline Silica polyHIPE & Crude oil (18) & 7 \\
\hline $\begin{array}{l}\text { PLA/PCL } \\
\text { polyHIPE }\end{array}$ & Gasoline $(\sim 2.5)$, diesel $(\sim 2.6)$ and vegetable oil $(\sim 2.2)$ & 8 \\
\hline PS polyHIPE & $\begin{array}{l}\text { Hexane (26.2), chloroform (62.0), toluene (37.4), diesel } \\
\text { (36.4) and edible oil (42.1) }\end{array}$ & 9 \\
\hline $\begin{array}{l}\text { HIPE-templated } \\
\text { porous sPS }\end{array}$ & $\begin{array}{l}\text { Chloroform ( } 81.3) \text {, dichloroethane }(66.0) \text {, toluene }(52.8) \text {, } \\
\text { hexane (33.9), vegetable oil }(45.0) \text {, olive oil }(44.4) \text {, petrol } \\
\text { (35.7) and diesel (41.9) }\end{array}$ & $\begin{array}{l}\text { This } \\
\text { work }\end{array}$ \\
\hline
\end{tabular}

a Abbreviation: PBA: poly(butyl acrylate); PBMA: poly(tertiary butylmethacrylate);

PCL: Poly( $\varepsilon$-caprolactone); PLA: Poly(1-lactide); PS: polystyrene. 


\section{References}

(1) Wu, Y.; Zhang, T.; Xu, Z.; Guo, Q. High Internal Phase Emulsion (HIPE) Xerogels for Enhanced Oil Spill Recovery. J. Mater. Chem. A 2015, 3, 1906-1909.

(2) Zhang, T.; Guo, Q. Continuous Preparation of PolyHIPE Monoliths from Ionomer-Stabilized High Internal Phase Emulsions (HIPEs) for Efficient Recovery of Spilled Oils. Chem. Eng. J. 2017, 307, 812-819.

(3) Chen, X.; Liu, L.; Liu, K.; Miao, Q.; Fang, Y. Facile Preparation of Porous Polymeric Composite Monoliths with Superior Performances in Oil-Water Separation - a Low-Molecular Mass Gelators-Based Gel Emulsion Approach. $J$. Mater. Chem. A 2014, 2, 10081-10089.

(4) Chen, X.; Liu, L.; Liu, K.; Miao, Q.; Lü, Y.; Fang, Y. Compressible Porous Hybrid Monoliths: Preparation via a Low Molecular Mass Gelators-Based Gel-Emulsion Approach and Exceptional Performances. J. Mater. Chem. A 2015, $3,24322-24332$.

(5) Guo, Z.; Gu, H.; Chen, Q.; He, Z.; Xu, W.; Zhang, J.; Liu, Y.; Xiong, L.; Zheng, L.; Feng, Y. Macroporous Monoliths with PH-Induced Switchable Wettability for Recyclable Oil Separation and Recovery. J. Colloid Interface Sci. 2019, 534, 183-194.

(6) Lee, J.; Chang, J. Y. Pickering Emulsion Stabilized by Microporous Organic Polymer Particles for the Fabrication of a Hierarchically Porous Monolith. Langmuir 2018, 34, 11843-11849. 
(7) Mahadik, D. B.; Lee, K.-Y.; Ghorpade, R. V.; Park, H.-H. Superhydrophobic and Compressible Silica-PolyHIPE Covalently Bonded Porous Networks via Emulsion Templating for Oil Spill Cleanup and Recovery. Sci. Rep. 2018, 8, 16783.

(8) Pérez-García, M. G.; Gutiérrez, M. C.; Mota-Morales, J. D.; Luna-Bárcenas, G.; del Monte, F. Synthesis of Biodegradable Macroporous Poly(l-lactide)/Poly( $\varepsilon$-caprolactone) Blend Using Oil-in-Eutectic-Mixture High-Internal-Phase Emulsions as Template. ACS Appl. Mat. Interfaces 2016, 8, 16939-16949.

(9) Zhang, N.; Zhong, S.; Zhou, X.; Jiang, W.; Wang, T.; Fu, J. Superhydrophobic P(St-DVB) Foam Prepared by the High Internal Phase Emulsion Technique for Oil Spill Recovery. Chem. Eng. J. 2016, 298, 117-124. 